\title{
HIGHLIGHTS OF ASTRONOMY
}

\section{VOLUME 13}

As presented at THE XXV $V^{T H}$ GENERAL ASSEMBLY of the IAU - 2003

Edited by: O. ENGVOLD
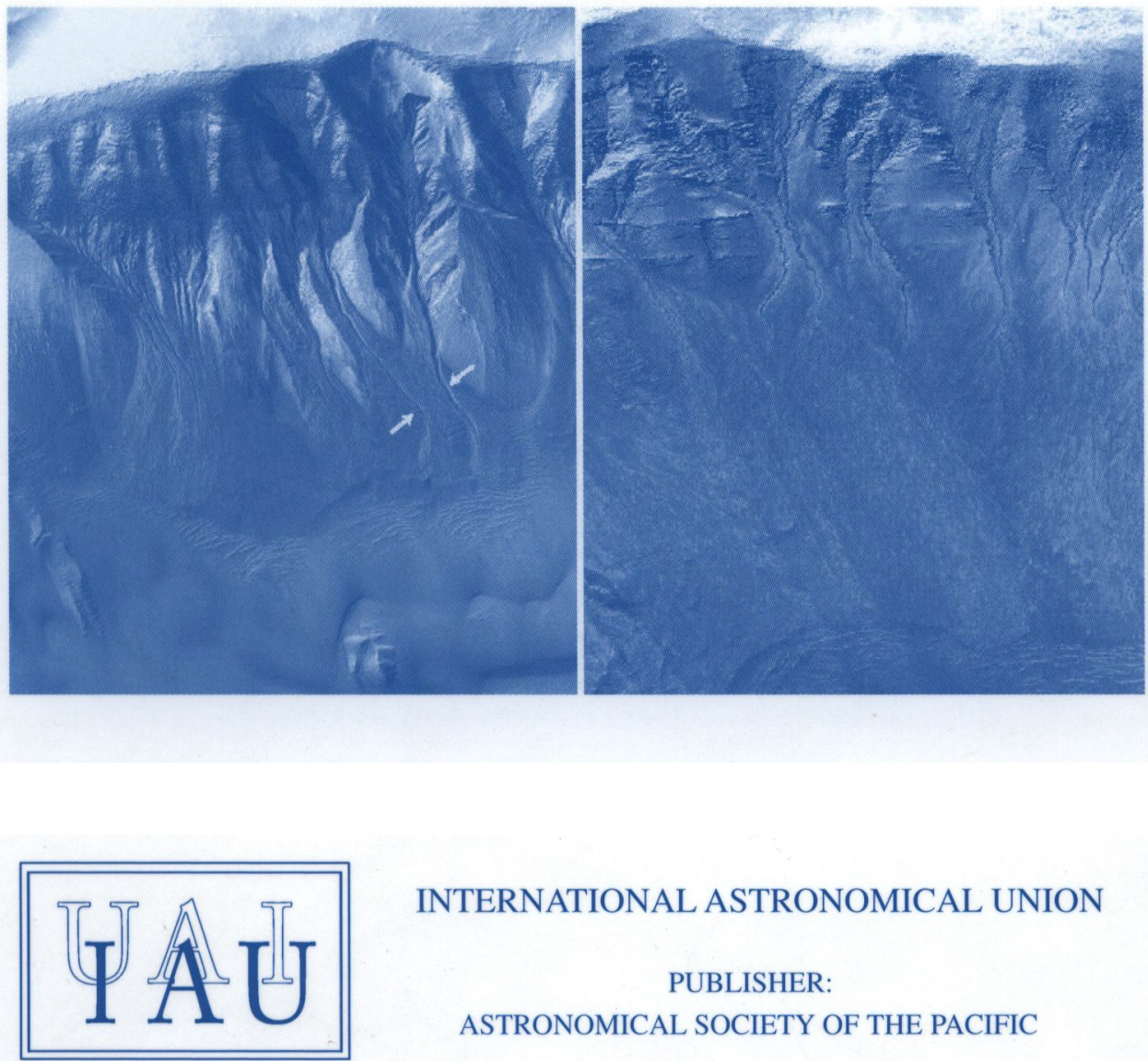

INTERNATIONAL ASTRONOMICAL UNION

PUBLISHER:

ASTRONOMICAL SOCIETY OF THE PACIFIC 


\section{HIGHLIGHTS OF ASTRONOMY}

Volume 13

COVER ILLUSTRATION:

High resolution image from the Mars Global Surveyor showing what may be flows from water at small depths below the level of the surrounding plains.

Reference: L. V. Ksanfomality, this volume,"Paradox of Flows on Mars", figure 1, page 918. 


\section{ASTRONOMICAL SOCIETY OF THE PACIFIC \\ 390 Ashton Avenue - San Francisco-California - USA 94112-1722 \\ Phone: (415) 337-1100 \\ E-Mail: service@astrosociety.org \\ Fax: (415) 337-5205 \\ Web Site: www.astrosociety.org}

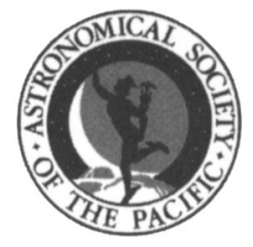

ASP-CS VOLUMES \& IAU PUBLICATIONS - EDITORIAL STAFF

Managing Editor: J. W. Moody

Publication Manager: Enid L. Livingston

PO Box 4666, Room N221 - ESC, Brigham Young University, Provo, Utah, 84602-4666

Phone: (801) 422-2111 Fax: (801) 422-0553 E-Mail: aspcs@byu.edu

LaTeX-Computer Consultant: T. J. Mahoney (Spain)-tjm(a)iac.es

A listing is cited at the back of this book of all IAU Volumes published by the

Astronomical Society of the Pacific Conference Series 


\title{
INTERNATIONAL ASTRONOMICAL UNION 98bis, Bd Arago - F-75014 Paris - France \\ Tel: +33143258358 E-mail: iau@iap.fr \\ Fax: +33 143252616 Web Site: www.iau.org
}

\section{HIGHLIGHTS OF ASTRONOMY}

\author{
VOLUME 13
}

AS PRESENTED AT

THE XXV ${ }^{\mathrm{TH}}$ GENERAL ASSEMBLY OF THE IAU - 2003

Edited by 
(C) 2005 by International Astronomical Union All Rights Reserved

No part of the material protected by this copyright notice may be reproduced or utilized in any form or by any means - graphic, electronic, or mechanical including photocopying, taping, recording or by any information storage and retrieval system, without written permission from the $I A U$.

Library of Congress Cataloging in Publication Data

Main entry under title

ISSN: $\quad 1539-2996$

ISBN : $\quad 1-58381-189-3$

IAU Publications - First Edition

Published on behalf of the IAU by: Astronomical Society of the Pacific

Printed in United States of America by Sheridan Books, Ann Arbor, Michigan 


\section{TABLE OF CONTENTS}

\section{Contents}

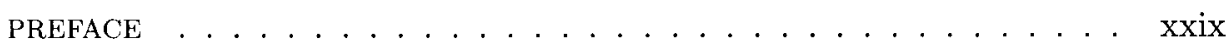

Oddbjorn Engvold

\section{JOINT DISCUSSIONS}

\section{NON-ELECTROMAGNETIC WINDOWS FOR ASTROPHYSICS}

Chairperson: $M$. Salvati

Editors: P. Blasi and M. Salvati

Neutrino Properties from Measurements using Astrophysical and

Terrestrial Sources . . . . . . . . . . . . . . . . .

A. B. McDonald

MeV Neutrino Sources: The Sun and the Supernovae . . . . . . . . 9

Sylvaine Turck-Chize

High-Energy Neutrino Astronomy: From AMANDA to IceCube . . . . .

Francis Halzen

Neutrinos from Pulsar Environments . . . . . . . . . . . . . . . .

A. Melatos

Some Aspects of Galactic Cosmic Ray Acceleration

Yousaf Mahmood Butt

Overview of Interferometer-Type Gravitational Wave Detectors . . . . . 30

David E. McClelland

A Window on the Ultra-High Energy Universe . . . . . . . . . . . 34

Pasquale Blasi

Experimental Progress in the Direct Detection of Dark Matter . . . . .

Nigel J. T. Smith

Dark Matter: Astronomical Aspects . . . . . . . . . . .

Virginia Trimble

Concluding Remarks: Non-Electromagnetic Windows in Astrophysics 45

Catherine J. Cesarsky 


\section{MERCURY}

Chairpersons:- R. Schulz and N. Thomas

Editors: R. Schulz, N. Thomas, and A. Sprague

Recent Advances in Ground-Based Observation of Mercury . . . . . . . 49

J. Warell

SpeX Spectroscopy of Mercury: $0.8-5.2 \mu \mathrm{m}$

Ann L. Sprague, Johan Warell, Joshua Emery, Angela Long,

John Rayner, and Mike Cushing

The Surface-Bounded Exosphere of Mercury . . . . . . . . . . . . . 56

Andrew Potter

Recycling of Ions in Mercury's Magnetosphere . . . . . . . . . . . . 60

Rosemary Killen, Andrew Potter, Menelaos Sarantos,

and Patricia Reiff

An Accurate Model of Mercury's Spin-Orbit Motion . . . . . . . . . . . 64

Nicola Rambaux and Eric Bois

Plasma Dynamics in Mercury's Magnetosphere . . . . . . . . . . . .

Wing-Huen Ip

Space Studies of the Black-Drop Effect at a Mercury Transit . . . . . .

Glenn Schneider, Jay M. Pasachoff, and Leon Golub

Origin and Bulk Chemical Composition of Mercury . . . . . . . . . .

Andrew J. R. Prentice and Daniel Jontof-Hutter

The BepiColombo Mission . . . . . . . . . . . . . . . . . . .

Rita Schulz, Peter Falkner, Anthony Peacock, Christian Erd,

Nicola Rando, and Stefan Kraft

The BepiColombo Lander-MSE

Nicolas Thomas, Rita Schulz, and Peter Falkner

\section{MAGNETIC FIELD AND HELICITY IN THE SUN AND HELIOSPHERE}

Chairpersons: B. Schmieder and D. M. Rust

Editors: B. Schmieder and D. M. Rust

Magnetic Helicity Conservation . . . . . . . . . . . . . . 85

Mitchell A. Berger

Helicity Generation and Signature in Solar Atmosphere . . . . . . . .

A. A. Pevtsov

Magnetohydrodynamic 3-D Models of the Solar Convection Zone . . . .

Allan Sacha Brun

Helicity and The Alpha-Effect: Dynamo Theory and Observations . . .

Kirill Kuzanyan 
Magnetic Helicity Propagation from Inside the Sun . . . . . . . . . .

Dana Longcope

Ejection of Bi-Helical Fields from the Sun

Axel Brandenburg and Eric G. Blackman

Magnetic Helicity in Sigmoids, Coronal Mass Ejections and Magnetic

Clouds . . . . . . . . . . . . . . . . .

D. M. Rust

Technique for Inferring Magnetic Helicity of Active Regions . . . . . . .

Jongchul Chae and Yong-Jae Moon

Generation and Annihilation of Magnetic Helicity in Active Regions . .

K. Kusano

Transport of Magnetic Helicity and Dynamics of Solar Active Regions .

M. K. Georgoulis, B. J. LaBonte, and D. M. Rust

Magnetic Field and Helicity in Solar Active Regions . . . . . . . . .

Hongqi Zhang

Twist and Writh of $\delta$ Active Region Magnetic Fields . . . . . . . . .

Lirong Tian and Jing Yang

Solar and Interplanetary Magnetic Helicity Balance of Active Regions

Cristina H. Mandrini, Pascal Démoulin, Lidia van Driel-Gesztelyi,

Sergio Dasso, Lucinda M. Green, and Marcelo López Fuentes

Heating the Solar Corona . . . . . . . . . . . . . . .

L. C. Woods

Helicity Pattern of CME Source Active Regions . . . . . . . . . . .

Jingxiu Wang, Guiping Zhou, and Jun Zhang

Helicity and the SOLIS Vector Spectromagnetograph . . . . . . . . .

Christoph U. Keller, John W. Harvey, Carl J. Henney, and

Harrison P. Jones

Prominence Formation Processes . . . . . . . . . . . . .

B. T. Welsch, C. R. DeVore, and S. K. Antiochos

The Role of Magnetic Helicity in Solar Flares . . . . . . . . . . . . . .

Mark G. Linton

Helicity of Magnetic Clouds and Their Associated Active Regions . . . .

Robert J. Leamon, Richard C. Canfield, Sarah L. Jones, Keith Lambkin,

Brian J. Lundberg, and Alexei A. Pevtsov

New Force-Free Models of Magnetic Clouds . . . . . . . . . . . . . .

M. Vandas, E. P. Romashets, and S. Watari

Magnetic Helicity Generated together with the Evolution of the

Large-Scale Magnetic Fields . . . . . . . . . . . . . . . . .

Pavel Ambrož 
Of Tilt and Twist . . . . . . . . . . . . . . . . . .

Zachary A. Holder, Richard C. Canfield, Rebecca A. McMullen,

Robert F. Howard, and Alexei A. Pevtsov

A Non-Helical Dynamo-MHD Simulations of Dynamo Action by a

Non-Helical Flow

V. Archontis and S. B. F. Dorch

RATAN-600 Observations of Unusual Inversion of Polarization in Sunspot Associated Microwave Sources . . . . . . . . . . . . . . . .

Nectaria A. B. Gizani, C. Alissandrakis, V. Bogod, V. Garaimov,

$V$. Zheleznyakov, and E. Zlotnik

Magnetic Neutral Line Rotations in Flare-Productive Regions . . . . .

Takako T. Ishii, Ayumi Asai, Hiroki Kurokawa, and Tsutomu T. Takeuchi

Diagnosis of Faraday Rotation with the Video Vector Magnetograph

at Huairou . . . . . . . . . . . . . . . . . . . . .

Jiangtao Su and Hongqi Zhang

On a Cyclic Variation of the Hemispheric Helicity Rule

A. A. Pevtsov, M. J. Hagyard, Z. Blehm, J. E. Smith, R. C. Canfield,

T. Sakurai, and M. Hagino

The Search for Correlation between BiSON SMMF Data and CME Events

William J. Chaplin, Andrew M. Dumbill, Yvonne P. Elsworth,

George R. Isaak, Clive P. McLeod, Brek A. Miller, Roger New, and Balázs Pintér

Eruption of a Quiescent Filament on February 18, 2003

Xingming Bao and Hongqi Zhang

The Current Helicity Parameter, $H_{\mathrm{c}}$, is More Sensitive than $\alpha_{\text {best }}$

to Faraday Rotation . . . . . . . . . . . . . . . . . .

Shudong Bao

\section{ASTROPHYSICAL IMPACT OF ABUNDANCES IN GLOBULAR CLUSTERS}

Chairpersons: F. D'Antona and R. Gratton

Editors: F. D'Antona and G. Da Costa

Summaries of Papers Presented at Joint Discussion Session 4:

Astrophysical Impact of Abundances in Globular Cluster Stars . . 147

G. S. Da Costa, F. D'Antona, and R. G. Gratton

Contributed Papers for JD4: Abstracts

\section{WHITE DWARFS: GALACTIC} AND COSMOLOGICAL PROBES

Chairperson: H. Shipman

Editors: $H$. Shipman and E. M. Sion 


\section{EXTRAGALACTIC GLOBULAR CLUSTERS AND THEIR HOST GALAXIES}

Chairpersons: T. Bridges and D. Forbes

Editors: T. Bridges and D. Forbes

M31's Disk System of Globular Clusters . . . . . . . . . . . . .

Heather Morrison, Paul Harding, Denise Hurley-Keller, and Kathy Perrett

The Evolution of NGC 5128: Globular Clusters and Field Stars . . . . .

Eric W. Peng

The Dynamical Mass of the Young Cluster W3 in NGC 7252:

Heavy-Weight Globular Cluster or Ultra-Compact Dwarf Galaxy?

Claudia Maraston, N. Bastian, R. P. Saglia, Markus Kissler-Patig, François Schweizer, and Paul Goudfrooij

Extreme Globular Cluster Systems . . . . . . . . . . . . . . .

John P. Blakeslee

Globular Clusters in Early-Type Galaxies with GMOS . . . . . . . . . .

Terry Bridges, Mike Beasley, Favio Faifer, Duncan Forbes, Juan Forte,

Karl Gebhardt, Dave Hanes, Ray Sharples, and Steve Zepf

Intergalactic Globular Clusters . . . . . . . . . . . . . . .

Michael J. West, Patrick Côté, Henry C. Ferguson, Michael D. Gregg,

Andrés Jordán, Ronald O. Marzke, Nial R. Tanvir, and Ted von Hippel

A Tale of Giants Stealing from Dwarfs . . . . . . . . . . . . . .

Duncan A. Forbes

The ACS Virgo Cluster Survey . . . . . . . . . . . . . . . .

Patrick Côté and Michael J. West

The Fundamental Plane of Globular Clusters . . . . . . . . . . . .

Dean E. McLaughlin

HST Observations of Young Stellar Clusters in Nearby Galaxies . . . .

Søren S. Larsen

Star Cluster Formation in Extreme Starburst Environments . . . . . . .

Richard de Grijs

From Young to Old: Spectral Models for Star Cluster Systems . . . . .

Uta Fritze-v. Alvensleben, Peter Anders, and Richard de Grijs

Stellar Population Models with Variable Element Abundance Ratios . .

Daniel Thomas, Claudia Maraston, and Ralf Bender

Formation of Globular Clusters in Galaxy Mergers . . . . . . . . . . .

Kenji Bekki, Warrick J. Couch, Duncan A. Forbes, and M. A. Beasley

Dynamical Evolution of Globular Cluster Systems . . . . . . . . .

E. Vesperini 
Multi-Color Observations of Young Star Clusters . . . . . . . . . .

Peter Anders, Uta Fritze - v. Alvensleben, and Richard de Grijs

Formation of $\omega$ Centauri from an Ancient Nucleated Dwarf Galaxy . . .

Kenji Bekki and K. C. Freeman

Dynamical Evolution of Globular Cluster Systems in Clusters of Galaxies:

The Case of NGC 1404 in the Fornax Cluster . . . . . . . . . 197

Kenji Bekki, Warrick J. Couch, Duncan A. Forbes, and M. A. Beasley

Formation of Star Clusters in the LMC and SMC . . . . . . . . . . . .

Kenji Bekki, Warrick J. Couch, Duncan A. Forbes, and M. A. Beasley

2dF Spectroscopy of Globular Clusters in M104 . . . . . . . . . . . . . .

Terry Bridges, Steve Zepf, Katherine Rhode, and Ken Freeman

Abundances in LMC and SMC Globular Clusters . . . . . . . . . . . .

Jennifer A. Johnson, Inese I. Ivans, Peter B. Stetson, James E. Hesser, and Michael Bolte

Perhaps They are not Globular Clusters After All

A. M. Karick

Self-Induced Formation of Metal-Rich Globulars in Bulges? . . . . . . .

Valery V. Kravtsov

The Spectra of Bright Near-IR Clusters in M82 . . . . . . . . . .

A. Lançon, M. Mouhcine, E. Chané, L. J. Smith, J. S. Gallagher,

N. Förster Schreiber, W. Vacca, R. de Grijs, and R. W. O'Connell

Evolution of Globular Cluster Populations in Compact Galaxy Groups .

S. Leon, J. Perea, A. Del Olmo, E. Athanassoula, G. Bergond,

G. Meylan, and C. Garcia Gomez

Globular Cluster Formation in Galaxy Mergers . . . . . . . . . . . . . .

Yuexing Li, Mordecai-Mark Mac Low, and Ralf S. Klessen

Multicolor Photometry and Age Estimates of Globular Clusters in M31

Jun Ma

Search for Formation Criteria for Globular Cluster Systems . . . . . . .

S. N. Nuritdinov, K. T. Mirtadjieva, and I. U. Tadjibaev

Young Stellar Clusters in the ULIRG IRAS 17208-0014 . . . . . . . . .

Naveen A. Reddy, Shardha Jogee, and Nick Scoville

SUBARU/FOCAS Globular Clusters Survey around M82 . . . . . . . .

Yoshihiko Saito, Masanori Iye, Nobunari Kashikawa, Koji S. Kawabata, Michitoshi Yoshida, Youichi Ohyama, Toshiyuki Sasaki, Tadafumi

Takata, George Kosugi, and Kentaro Aoki

Chemical Abundances in the Sagittarius Galaxy: Terzan 7 . . . . . . . .

Gražina Tautvaišiene, George Wallerstein, Doug Geisler,

Guillermo Gonzalez, and Corinne Charbonnel 


\section{THE SUN AND THE HELIOSPHERE}

\section{AS AN INTEGRATED SYSTEM}

Chairpersons: G. Poletto and S. T. Suess

Editors: G. Poletto and S. T. Suess

JD 7: The Sun and the Heliosphere as an Integrated System . . . . . 213

Giannina Poletto and Steven T. Suess

\section{LARGE TELESCOPES AND VIRTUAL OBSERVATORY: VISIONS FOR THE FUTURE}

Chairpersons: F. Genova and Ding-qiang Su

Editors: F. Genova and Xiangqun Cui

Joint Discussion 8: Large Telescopes and Virtual Observatories:

Visions for the Future . . . . . . . . . . . . . . . . 245

Françoise Genova and Cui Xiangqun

\section{ASTROTOMOGRAPHY}

Chairpersons: M. Richards and L. Morales Rueda

Editors: A. Collier Cameron, A. Schwope, and S. Vrielmann

Joint Discussion 9: Astrotomography . 279

Andrew Collier Cameron, Axel Schwope, and Sonja Vrielmann

\section{EVOLUTION IN GALAXY CLUSTERS:}

\section{A MULTIWAVELENGTH APPROACH}

Chairpersons: L. Feretti and R.W. Hunstead

Editors: R. W. Hunstead, L. Feretti, B. Gibson, and P. Nulsen

Evolution in Galaxy Clusters: A Multiwavelength Approach . . . . . . . 285

Dick Hunstead and Luigina Feretti

Clusters in the Optical . . . . . . . . . . . . . . . . . . 286

Lori M. Lubin

Mergers and Non-Thermal Processes in Clusters . . . . . . . . . . . . . 291

Craig L. Sarazin

Stellar Populations, Butcher-Oemler Effect, Star Formation in Clusters

Bianca M. Poggianti

Radio Sources as Probes of Distant Clusters . . . . . . . . . . . . . . . 302

Joanne Baker, Jordi Barr, and Malcolm Bremer

X-Ray Cavities and Cooling Flows . . . . . . . . . . . . . . . . 307

Paul E. J. Nulsen, Brian R. McNamara, Laurence P. David, and Michael W. Wise 
Radio Halo and Relic Sources in Galaxy Clusters . . . . . . . . . . . . 312

Kinwah Wu, Melanie Johnston-Hollitt, and Richard Hunstead

Non-Thermal Activity and Particle Acceleration in Clusters of Galaxies

Vahe' Petrosian

Magnetic Fields in Galaxy Clusters . . . . . . . . . . . . . . . .

Federica Govoni and Matteo Murgia

Contributed Papers for JD10: Abstracts . . . . . . . . . . . . .

\section{DYNAMICS AND EVOLUTION \\ OF DENSE STELLAR SYSTEMS}

Chairpersons: F. Combes and D. Richstone

Editors: F. Combes, P. Hut, and D. Richstone

MODEST: Modeling Stellar Evolution and (Hydro)Dynamics . . . . . .

Piet Hut

Black Hole Binary Mergers $\ldots \ldots \ldots \ldots \ldots$

Junichiro Makino

Can Bars Be Destroyed by Central Mass Concentrations?

E. Athanassoula, W. Dehnen, and J. C. Lambert

The Formation and Evolution of Star Clusters and Galaxies . . . . . .

Stephen E. Zepf

Formation and Evolution of Massive Black Holes in Star Clusters . . . .

Holger Baumgardt, Junichiro Makino, and Simon Portegies Zwart

A Physicist's View of Stellar Dynamics: Dynamical Instability of Stellar

Systems . . . . . . . . . . . . . . . . .

V. G. Gurzadyan

Formation of Young Star Clusters . . . . . . . . . . . . . 358

Bruce Elmegreen

"Super" Star Clusters . . . . . . . . . . . . . . . . . . . .

Richard de Grijs

Young Star Clusters: Progenitors of Globular Clusters!? . . . . . . . . .

Peter Anders, Uta Fritze - v. Alvensleben, and Richard de Grijs

A New Scenario for the Formation of Massive Stellar Clusters . . . . . .

Jan Palouš, Guillermo Tenorio-Tagle, Sergiy Silich,

Gustavo A. Medina-Tanco, and Casiana Muñoz-Tuñon

Mass Loss, Kinematics, and the Evolution of Super Star Clusters in

the Antennae . . . . . . . . . . . . . . . .

Andrea M. Gilbert and James R. Graham

Contributed Papers for JD11: Abstracts . . . . . . . . . . . . 


\section{SOLAR AND SOLAR-LIKE OSCILLATIONS: INSIGHTS AND CHALLENGES FOR THE SUN AND STARS}

Chairpersons: T. R. Bedding and J. Leibacher

Editors: T. R. Bedding and J. Leibacher

Asteroseismology: From Dream to Reality . . . . . . . . . . . . . . . 391

D. W. Kurtz

Physics of Solar-Like Oscillations . . . . . . . . . . . . . . . . 397

Jørgen Christensen-Dalsgaard

Challenges in Stellar Models from Helioseismology to Asteroseismology

Sylvaine Turck-Chièze, Phu Anh Phi Nghiem, and Laurent Piau

Observations of Solar-like Oscillations . . . . . . . . . . . . . . 407

Hans Kjeldsen and Timothy R. Bedding

Excitation of P-Modes in the Sun and Stars . . . . . . . . . . . .

Robert Stein, Dali Georgobiani, Regner Trampedach, Hans-Günter

Ludwig, and Åke Nordlund

Helio- and Asteroseismic Analysis Methods . . . . . . . . . . . . . . . 415

Jesper Schou

Observational Results of Full-Disc Helioseismology . . . . . . . . . .

Roger New

Seismology of Solar Internal Rotation . . . . . . . . . . . . . . 424

Takashi Sekii

Local Helioseismology-What Does It Really Tell Us? . . . . . . . . . . 428

Frank Hill

Solar Meridional Flows: Recent Findings

Deborah A. Haber and Bradley W. Hindman

Observations and Interpretation of Subsurface Magnetic Structures . . .

P. S. Cally and A. D. Crouch

\section{EXTRAGALACTIC BINARIES}

Chairperson: I. Ribas and A. Giménez

Editor: I. Ribas and A. Gimenez

Joint Discussion 13: on Extragalactic Binaries . . . . . . . . . . 441 Ignasi Ribas and Alvaro Giménez

Seeing Double in the Local Group: Extragalactic Binaries . . . . . . . 446

Edward F. Guinan

Binary Stars in the Local Group: The Playing Field

Mario L. Mateo 
A Review of the Distance and Structure of the Large Magellanic Cloud

David R. Alves

Binary Star Research Using the MACHO Database . . . . . . . . . . . 450

Kem H. Cook

Eclipsing Binaries in the Magellanic Clouds . . . . . . . . . . . . . . .

Andrzej Udalski

DIRECT DEBs in M31 and M33

Lucas M. Macri

Eclipsing Binaries as Precise Standard Candles and Distance Indicators

Jens Viggo Clausen

Eclipsing Spectroscopic Binaries in the SMC . . . . . . . . . . . . .

Ron W. Hilditch, Tim J. Harries, and Ian D. Howarth

Semi-Detached Binaries as Probes of the Local Group . . . . . . . . . . 456

Robert E. Wilson

Contact Binaries of the W UMa Type as Distance Tracers . . . . . . . .

Slavek M. Rucinski

Cepheid Variables in Ecliping Binary Systems

Douglas Welch

Improved Light Curves of LMC Eclipsing Binaries . . . . . . . . . . .

Young Woon Kang, Kyungsoo Hong, Woo-Baik Lee, Ho-Il Kim,

and Kyu-Dong Oh

Detached Binaries in the Large Magellanic Cloud . . . . . . . . . . . . .

G. Michalska and A. Pigulski

Massive Binaries in the Magellanic Clouds . . . . . . . . . . . . . . .

Virpi S. Niemela

Extragalactic Eclipsing Binaries: Astrophysical Laboratories

Ignasi Ribas

Circularization in B-Bype Eclipsing Binaries in both Magellanic Clouds

P. North and J.-P. Zahn

Orbital Elements of MACHO Project Eclipsing Binary Stars . . . . .

Charles Alcock

Review of SN Ia Progenitors

Brian P. Schmidt

Extragalactic Binaries as Core-Collapse Supernova Progenitors . . . . . 469

Schuyler D. Van Dyk 


\section{FORMATION OF COMETARY MATERIAL}

Chairperson: W. F. Huebner

Editor: W. F. Huebner, P. Ehrenfreund, and H.-U. Keller

Apparent Inconsistencies in the Formation of Cometary Matter . . . . . 473

W. F. Huebner and H. U. Keller

Large Interstellar and Cometary Biomolecules . . . . . . . . . . . 476

Lewis E. Snyder

Processed and Unprocessed Ices in Circumstellar Disks . . . . . . . . . 479

Klaus Pontoppidan, Ewine van Dishoeck, Emmanuel Dartois,

and Wing-Fai Thi

Ortho-to-Para Ratio of Cometary Water and Ammonia . . . . . . . . 482

Hideyo Kawakita, Jun-ichi Watanabe, Reiko Furusho, and Tetsuharu Fuse

Material Processing of Interstellar Dust in Comets . . . . . . . . . . .

Hiroshi Kimura and Ingrid Mann

From Interstellar Matter to Comets: A Laboratory View . . . . . . . . .

Pascale Ehrenfreund and Oliver Botta

Implications of Ice Morphology for Comet Formation . . . . . . . . .

M. P. Collings, J. W. Dever, M. R. S. McCoustra, and H. J. Fraser

Cometary Silicates: Interstellar and Nebular Materials . . . . . . . . .

Diane H. Wooden

Light Scattering as a Clue to Cometary Dust Structure . . . . . . . . 498

A. Chantal Levasseur-Regourd, E. Hadamcik, and J. Lasue

The Nature of Diatomic Sulfur in Comets . . . . . . . . . . . . . 501

D. C. Boice and Céline Reylé

Solar Composition Icy Planetesimals: A New Source for Comet Nuclei? 502 Tobias Owen

Models of Collapsing Clouds and Star-Forming Regions as Analogs of the Solar Nebula . . . . . . . . . . . . . . . . . 504

Paola Caselli

The Structure of the Solar Nebula from Cometary Composition . . . . . 508

D. Bockelée-Morvan, F. Hersant, D. Gautier, and J.-M. Huré

Chemistry of Collapse and Disk Accretion . . . . . . . . . . .

S. B. Charnley and S. D. Rodgers

Chemistry in Protoplanetary Disks . . . . . . . . . . . .

Yuri Aikawa

Disk Chemistry and Cometary Composition . . . . . . . . . . 518

A. J. Markwick and S. B. Charnley 
A Mechanism of Crystallization of Cometary Silicates . . . . . . . . . . 522

Tetsuo Yamamoto and Takeshi Chigai

Crystallization of Silicate Particles by Shock Waves . . . . . . . . . 525

Taishi Nakamoto and Hitoshi Miura

Collisional Simulations of Cometary Nuclei . . . . . . . . . . . . . . 528

Sin-iti Sirono

Impacts onto Cometary Nuclei . . . . . . . . . . . . . . . . . . 531

Jacek Leliwa-Kopystynski

\section{ELEMENTAL ABUNDANCES IN OLD STARS \& DAMPED LYMAN-ALPHA SYSTEMS}

Chairpersons: P.E. Nissen and M. Pettini

Editors: P.E. Nissen and M. Pettini

Introduction to Joint Discussion 15: 'Elemental Abundances in Old Stars and Damped Lyman-Alpha Systems' . . . . . . . . . . . . 535 Poul E. Nissen and Max Pettini

Comparing Chemical Abundances of the Damped Ly $\alpha$ Systems and Metal-Poor Stars . . . . . . . . . . . . . 536 Jason X. Prochaska

Uncertainties in Stellar Abundance Analyses . . . . . . . . . . . . . . . 542 Martin Asplund

Stellar Abundances in Local Group Galaxies . . . . . . . . . . . . . . . 548 Eline Tolstoy and Kim Venn

The Early Chemical Evolution of Dwarf Irregular Galaxies . . . . . . . 554 Gerhard Hensler, Simone Recchi, Joachim Köppen, and Andreas Rieschick

Nucleosynthesis in Population III Supernovae . . . . . . . . . . . . . 560

K. Nomoto, K. Maeda, H. Umeda, and N. Tominaga

Evolution of Metals and Stars in Damped Lyman-Alpha Galaxies . . . . 566 Varsha P. Kulkarni

The Star Formation History of Damped Lyman-Alpha Systems . . . . . 572 Arthur M. Wolfe

Contributed Papers for JD15: Abstracts . . . . . . . . . . . . 578 


\section{THE INTERNATIONAL CELESTIAL REFERENCE SYSTEM: MAINTENANCE AND FUTURE REALIZATION}

Chairpersons: D. McCarthy and F. Mignard

Editors: R. Gaume, D. McCarthy, and J. Souchay

Summary of IAU Joint Discussion 16: 'The International Celestial Reference System, Maintenance, and Future Realizations' . . . . .

Dennis D. McCarthy

Contributed Papers for JD16: Abstracts . . . . . . . . . . .

\section{ATOMIC DATA FOR X-RAY ASTRONOMY}

Chairperson: A. K. Pradhan

Editor: A. K. Pradhan, Sultana Nahar, and P. L. Smith

IAU XXV JD17: Atomic Data For X-Ray Astronomy . . . . . . . .

Anil K. Pradhan, Sultana N. Nahar, and Peter L. Smith

Probing X-ray Emitting Plasma with High Resolution Chandra and XMM-Newton Spectra . . . . . . . . . . . . .

Julia C. Lee

The First Results from the Solar X-ray Spectrometer (SOXS) Mission

Rajmal Jain, Hemant Dave, P. Sreekumar, A. B. Shah, N. M. Vadher,

K. S. B. Manian, G. P. Ubale, V. M. Shah, K. J. Shah, S. L. Kayasth,

V. D. Patel, Sumit Kumar, and M. R. Deshpande

The Iron Project and the $\mathrm{RmaX}$ Project . . . . . . . . . . . . .

Anil K. Pradhan

Atomic Physics Calculations for Iron L-Line Spectra

Jacques Dubau, Delphine Porquet, and Oleg Zabaydullin

Iron $\mathrm{K} \alpha$ Spectra from an Atomic Modeling Perspective

Duane A. Liedahl

New Results in Laboratory X-ray Astrophysics

P. Beiersdorfer, H. Chen, K. R. Boyce, G. V. Brown, R. L. Kelley,

F. S. Porter, C. K. Stahle, J. K. Lepson, J. G. Jernigan, B. J. Wargelin, and S. M. Kahn

Photorecombination and Photoionization Experiments at Heavy-Ion

Storage-Rings and Synchrotron-Light Sources . . . . . . . .

Stefan Schippers

The Ionized Gas and Nuclear Environment in NGC 3783 . . . . . . . .

Hagai Netzer

New Results on X-ray Models and Atomic Data

Jelle S. Kaastra, Rolf Mewe, and Ton Raassen 
xviii Contents

Spectral Modeling with APEC

Nancy S. Brickhouse and Randall K. Smith

The CHIANTI Database . . . . . . . . . . . . . . . . . . . .

E. Landi, K. P. Dere, P. R. Young, M. Landini, H. E. Mason,

and G. Del Zanna

Atomic Spectral Tables for the Chandra X-ray Observatory . . . . . .

L. I. Podobedova, D. E. Kelleher, J. Reader, and W. L. Wiese

TIPTOPbase . . . . . . . . . . . . . . . . . 662

P. Palmeri and C. Mendoza

The Astrophysical Plasma Emission Database: Progress and Plans . . . 666

Randall K. Smith, Nancy S. Brickhouse, and Duane A. Liedahl

New Radiative Atomic Data . . . . . . . . . . . . . . . . . 668

Sultana N. Nahar

NIFS Atomic Numerical Databases . . . . . . . . . . . . . . . . . 672

Izumi Murakami and Takako Kato

Future NASA Programs and Funding Support . . . . . . . . . . . 674

Hashima Hasan

Distinguishing Models for ACIS Data of Diffuse Emission . . . . . . .

D. A. Leahy

\section{QUASAR CORES AND JETS}

Chairperson: D.L. Jauncey

Editors: D. L. Jauncey, K. I. Kellermann, and J. V. Wall

X-Ray and Optical Properties of Radio Jets . . . . . . . . . . . . . 685

D. $M$. Worrall

The Cosmic Evolution of Quasars _. . . . . . . . . . . . . . . 692

Carole Jackson, Jasper Wall, Peter Shaver, Ken Kellermann, and Isobel Hook

Central Regions of AGNs Probed by Neutral Hydrogen . . . . . . . . . 698 Raffaella Morganti

Rapid Interstellar Scintillation of Quasar PKS 1257-326 . . . . . . . 703

Hayley E. Bignall, David L. Jauncey, James E. J. Lovell,

Anastasios K. Tzioumis, Jean-Pierre Macquart, and

Lucyna Kedziora-Chudczer

The Jets in Micro-Quasars and Quasars: A Comparison . . . . . . . . 709 Ralph Spencer

JD18: Quasar Cores and Jets-Poster-Paper Summary _....... 714 J. V. Wall 


\section{PHYSICAL PROPERTIES AND MORPHOLOGY OF SMALL SOLAR SYSTEM BODIES}

Chairpersons: H. U. Keller and E. F. Tedesco

Editors: E. F. Tedesco, W. F. Huebner, and H. U. Keller

Welcome and Introduction $\ldots \ldots \ldots \ldots \ldots \ldots$

Edward F. Tedesco

Galileo's Exploration of Small Bodies

Torrence Johnson

NEAR at Mathilde and Eros: An Update

Andrew Cheng

Comet Halley Observed during the ESA Giotto Fly-By $\ldots \ldots \ldots \ldots$

H. U. Keller

The Deep Space 1 Encounter With Comet 19P/Borrelly . . . . . . . .

Robert M. Nelson

Rosetta Asteroid Candidates

M. Antonietta Barucci, Marcello Fulchignoni, Mirel Birlan,

Pierre Vernazza, Elisabetta Dotto, and Alain Doressoundiram

Muses-C as a Benchmark Mission for S-Type Asteroid Group . . . . . .

Akira Fujiwara, Masanao Abe, and Hajime Yano

Dawn Discovery Mission: Symbiosis with 1 AU Observations . . . . .

C. T. Russell

Observations of Small Solar System Bodies with GAIA . . . . . . . . .

Francois Mignard

The ISHTAR Mission: Probing the Internal Structure of NEOs . . . .

M. Antonietta Barucci, Paolo D'Arrigo, P. Ball, Alain Doressoundiram,

Elisabetta Dotto, W. Kofman, Roberto Orosei, Martin Patzold, and Ettore Perozzi

A Target for Rosetta . . . . . . . . . . . . . . . . . . . . . . . . .

Rita Schulz

The Deep Impact Project . . . . . . . . . . . . . . . . . . .

Michael F. A'Hearn and the Deep Impact Project Team

ISO: Asteroid Results and Thermophysical Modelling

Thomas G. Müller

Ground-Based Optical Observations of Asteroids

Alberto Cellino

Extended Families in the Main Belt and in the Trojan Swarms

Zoran Knezevic and Andrea Milani

Radar Observations of Near-Earth Asteroids . . . . . . . . . . . .

Micael C. Nolan, Lance A. Benner, Greg Black, Don B. Campbell, Jon D. Giorgini, Alice A. Hine, Ellen S. Howell, Jean-Luc Margot, and Steven J. Ostro 
Minor Planet Binaries . . . . . . . . . . . . . . . . . . 760

Jean-Luc Margot

ISO Observations of Comets . . . . . . . . . . . . . . . . . . 761

Dominique Bockelée-Morvan

Comets .......................... 762

Michael F. A'Hearn

Radar Observations of Comet Nuclei and Comae . . . . . . . . . . 763

Donald B. Campbell, John K. Harmon, Micael C. Nolan, and Steven J. Ostro

Contributed Papers for JD19: Abstracts . . . . . . . . . . . . . . 764

\section{FRONTIERS OF HIGH RESOLUTION SPECTROSCOPY}

Chairperson: J. Linsky

Editor: J. Linsky

Introducing Joint Discussion 20: Frontiers of High Resolution Spectroscopy Jeffrey L. Linsky

Gamma-ray Spectroscopy

Jürgen Knödlseder

Astrophysics at X-Ray Spectral Resolution $1000 \ldots \ldots \ldots \ldots$

Nancy S. Brickhouse

High Resolution X-ray Spectroscopy: Is It Interesting? Is It Possible? . 790

Webster Cash

FUSE and the Quest for High-Resolution Spectroscopy in the Far

Ultraviolet . . . . . . . . . . . . . . . . . . 793

H. Warren Moos

APEX, the Astrophysical Plasmadynamic EXplorer: An EUV High

Resolution Spectroscopic Observatory . . . . . . . . . . 796

M. Kowalski

High Spatial/Spectral Resolution Studies of Eta Carinae . . . . . . . . 799

Theodore R. Gull and the Eta Carinae HST Treasury Team

The Future for UV Spectroscopy of the ISM at High Resolution . . . . . 804

Edward B. Jenkins

Mapping of Stellar Surfaces with Doppler and Zeeman Doppler Imaging

Andrew Collier Cameron

High-Resolution Optical Observations of Interstellar Absorption Lines .

Daniel E. Welty

The Deuterium Balmer Series . . . . . . . . . . . . . .

Guillaume Hébrard 
Surface Gravities and Masses in Substellar Objects . . . . . . . . . .

Subhanjoy Mohanty, Gibor Basri, and Ray Jayawardhana

Future Groundbased High-Resolution IR Spectrometers . . . . . . . . .

Alan Moorwood

High-Resolution Infrared Far-IR Spectroscopy from SOFIA, 2005-2025 .

J. A. Davidson and E. F. Erickson

Chemistry of Diffuse Clouds and Circumstellar Envelopes . . . . . . . .

Robert Lucas

Spectral-Line Surveys at Millimeter and Submillimeter Wavelengths:

The Impact of Spectral Resolution . . . . . . . . . . . . . . . .

L. M. Ziurys

High Resolution Spectroscopy at Low Radio Frequencies . . . . . . . . .

Jayaram N. Chengalur

\section{THE ASTROCHEMISTRY OF EXTERNAL GALAXIES}

Chairperson: T. J. Miller

Editor: T. J. Millar

Molecular Hydrogen in the High Redshift Damped Ly- $\alpha$ Systems . . . . 833

$R$. Srianand, P. Petitjean, C. Ledoux, and G. Ferland

Molecular Absorption Lines in Galaxies . . . . . . . . . . . . . . . . .

Tommy Wiklind

Deep Searches for High Redshift Molecular Absorption

S. J. Curran J. K. Webb, M. T. Murphy, and N. Kuno

Probing Physics and Chemistry in Circumnuclear Torus with $\mathrm{OH}$.... .

Yu Zhi-yao

Newly detected $\mathrm{H}_{2} \mathrm{O}$ Masers in Seyfert and Starburst Galaxies . . . . .

A. B. Peck, A. Tarchi, C. Henkel, N. M. Nagar, J. Braatz,

and $L$. Moscadelli

Molecular Line Observations in the Magellanic Clouds . . . . . . . . . .

Mónica Rubio

CO $J=7 \rightarrow 6$ Emission in the Large Magellanic Cloud

Sungeun Kim, Wilfred Walsh, Kecheng Xiao, Adair P. Lane, and Antony A. Stark

Magellanic Diffuse Interstellar Bands and Carbon Chemistry . . . . . .

P. Ehrenfreund, N. Cox, J. Cami, B. H. Foing, L. Kaper, L. d'Hendecourt,

J. P. Maier, F. Salama, P. Sarre, T. Snow, and P. Sonnentrucker

Young Star Clusters: Metallicity Tracers in External Galaxies . . . . . .

Peter Anders, Uta Fritze - v. Alvensleben, and Richard de Grijs

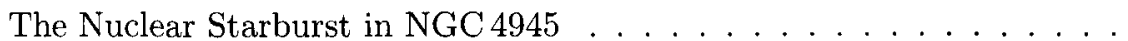

M. Wang, C. Henkel, Y.-N. Chin, J. B. Whiteoak, M. Hunt Cunningham, and $R$. Mauersberger 
A Gas and Dust Rich Giant Elliptical Galaxy . . . . . . . . . . .

$O$. Krause, U. Lisenfeld, U. Klaas, D. Lemke, M. Haas, and M. Stickel

Systematically Peculiar Molecular Composition in M 82: Regarding

the Formation Mechanisms . . . . . . . . . . . . . . . . . .

S. Takano, N. Nakai, K. Kawaguchi, T Takano, P. Schilke,

and $G$. Winnewisser

Extragalactic Ammonia

C. Henkel, K.M. Menten, J. Braatz, R. Mauersberger, A. Weiß,

M. Lebrón, A. Tarchi, A. B. Peck, C. L. Carilli, and D. A. Lubowich

\section{SPECIAL SCIENTIFIC SESSIONS}

\section{SPS 1. RECENT PROGRESS IN PLANETARY EXPLORATION}

Chairpersons: C. de Bergh and D. Cruikshank

Editors: D. Cruikshank and C. de Bergh

Jupiter after the Galileo Probe . . . . . . . . . . . . . . . . .

Richard E. Young and Tobias Owen

Volcanism on Io: The Post-Galileo View, and a Comparison with Earth Ashley Gerard Davies

New Results on the Composition of the Outer Planets and Titan . . . .

Thierry Fouchet

Comparative Planetary Atmospheres of the Galilean Satellites . . . .

Darrell F. Strobel

The Distribution and Nature of Titan's Aerosols: A New Look

Mark T. Lemmon, Peter H. Smith, and Ralph D. Lorenz

Characterization of the Zonal Wind Flow in the Upper Atmosphere of Titan with the VLT . . . . . . . . . . . . . . . .

Régis Courtin, David Luz, Daniel Gautier, Thierry Appourchaux, Jean-Pierre Lebreton, Francesca Ferri, Luisa Lara, Frédéric Hourdin, and Andreas Kaufer

The Abundant Irregular Satellites of the Giant Planets

Scott S. Sheppard and David C. Jewitt

Is Amalthea a Captured Trojan Asteroid of Jupiter? . . . . . . . . . . . 901

Andrew J. R. Prentice

Complex Organic Solid Matter in the Outer Solar System . . . . . . . 902

D. P. Cruikshank

Cassini/Huygens Mission to Saturn: Results and Prospects

Dennis L. Matson, Jean-Pierre Lebreton, and Linda Spilker

The Huygens Mission to Titan: Overview and Status . . . . . . . . . .

J.-P. Lebreton and D. L. Matson 
Changes in Pluto's Atmosphere . . . . . . . . . . . . . . . . 906

J. L. Elliot

Large Changes in Pluto's Atmosphere Revealed by Stellar Occultations 908

Bruno Sicardy, Thomas Widemann, Emmanuel Lellouch, Françoise

Roques, Eric Gendron, Christian Veillet, Jean-Charles Cuillandre,

François Colas, Wolfgang Beisker, Mike Kretlow, Olivier R. Hainaut, and Chris Lidman

The New Horizons Mission to Pluto/Charon and the Kuiper Belt . . . .

G. Leonard Tyler, S. Alan Stern, and Harold A. Weaver

Mapping Mars at Global to Human Scales . . . . . . . . . . . . . . .

Brent A. Archinal, Randolph L. Kirk, Elpitha Howington-Kraus,

Mark R. Rosiek, Laurence A. Soderblom, and Ella M. Lee

Planetary Exploration and Archaeology: Heritage Conservation . . . .

John B. Campbell

Numerical Simulation of the Jovian Wind Band as a Convective

Phenomenon . . . . . . . . . . . . . .

Kwing L. Chan and Hans G. Mayr

Charon/Pluto Light Ratio

K. B. Clancy, J. L. Elliot, and M. J. Person

Hot Hydrogen in the Jovian Corona . . . . . . . . . . . . . 917

C. Emerich, L. Ben Jaffel, J. T. Clarke, and G. Ballester

Paradox of Flows on Mars . . . . . . . . . . . . . . . . . . 918

L. V. Ksanfomality

ASTROD I: Mission Concept and Venus Flybys . . . . . . . . . . . . . 921

Guangyu Li, Wei-Tou Ni, and Chien-Jen Tang

Orbital Evolution of the Kuiper Belt . . . . . . . . . . . . . . . .

Charles Morgan and Andrew Prentice

Origin and Distribution of Water amongst the Inner Planets

Andrew J. R. Prentice

Winds in Venus' Lower Mesosphere.

Thomas Widemann and Emmanuel Lellouch

\section{SPS 2. ASTRONOMY IN ANTARCTICA}

Chairperson: $M$. Burton

Editor: $M$. Burton

The Potential for Astronomy in Antarctica

M. G. Burton

Particle Astronomy from Antarctica $\ldots \ldots \ldots \ldots \ldots$

Per Olof Hulth 
The 23 November 2003 Total Solar Eclipse in Antarctica . . . . . . . . . 931 Jay M. Pasachoff

Site Testing at Dome C-Cloud Statistics from the ICECAM Experiment 932 Michael C. B. Ashley, Michael G. Burton, Paolo G. Calisse, Andre Phillips, and John W. V. Storey

Millimetric Site Testing at Dome C: Results and Plans . . . . . . . . . .

Luca Valenziano, Giorgio Dall'Oglio, Andrea Graziani, Lorenzo Martinis, Gabriella Pizzo, and Lucia Sabbatini

Results from the South Pole Infra-Red EXplorer Telescope . . . . . .

J. M. Rathborne and M. G. Burton

The AST/RO Survey of the Galactic Center Region

Antony A. Stark

Antarctic Cosmic Ray Astronomy $\ldots \ldots \ldots \ldots \ldots$

Marc Duldig

IceCube: A Kilometer-Scale Neutrino Observatory at the South Pole . .

Francis Halzen

Beyond Dome $\mathrm{C}$

J. W. V. Storey, M. C. B. Ashley, M. G. Burton, and J. S. Lawrence

ACMSA: Antarctic Centre for Millimetre and Sub-millimetre

Astrophysics

Giorgio Sironi

The Case for a $30 \mathrm{~m}$ Diameter Submillimeter Telescope on the

Antarctic Plateau . . . . . . . . . . . . . . . . . .

Antony A. Stark

Extremely Large Telescopes on the Antarctic Plateau . . . . . . . . . .

J. S. Lawrence

A Large Reflective Schmidt Telescope for Antarctica . . . . . . . . . . . 958

Will Saunders and Andrew McGrath

Adaptive Optics and Interferometry on the Antarctic Plateau . . . . . .

James P. Lloyd

The Antarctic Planet Interferometer . . . . . . . . . . . . . . . . 962

Mark R. Swain

High Angular Resolution Mid-IR Astronomy at Concordia . . . . . . . . 964

Marco Ferrari-Toniolo

An AST/RO survey of the Coalsack . . . . . . . . . . . . . 965

Wilfred Walsh and Kecheng Xiao

Helioseismology from South Pole: Past, Present, and Future . . . . . . . 966

Stuart M. Jefferies

CO 2-1 Mapping of WR16 with AST/RO

N. F. H. Tothill, A. P. Marston, C. L. Martin, and K. Leppik 
History of Astrophysics in Antarctica-A Brief Overview

Balthasar T. Indermuehle, Michael G. Burton, and Sarah T. Maddison

CMB Observations from the Antarctic Plateau . . . . . . . . . . . .

G. Sironi, G. Boella, M. Gervasi, A. Passerini, A. Tartari,

and $M$. Zannoni

Earth as an Extrasolar Planet: South Pole Advantages . . . . . . . . .

Wesley A. Traub, Antony A. Stark, Kenneth W. Jucks, Steven Kilson,

Edwin L. Turner, and Sara Seager

The Explorer of Diffuse Galactic Emission (EDGE) . . . . . . . . . .

R. F. Silverberg, E. S. Cheng, D. A. Cottingham, D. J. Fixsen,

L. Knox, S. S. Meyer, P. T. Timbie, and G. W. Wilson

Compact Wide-Field Astronomical Telescopes for Dome C . . . . . . . .

Roberto F. Viotti and the TRT team

FTS Opacity Measurements of the South Pole Submillimeter Sky . . . .

Richard A. Chamberlin

\section{SPS 3. A NEW CLASSIFICATION SCHEME FOR DOUBLE STARS}

Chairpersons: W. I. Hartkopf and B. D. Mason

Editors: W. I. Hartkopf and B. D. Mason

An Introduction to the Nomenclature Problem . . . . . . . . . . . 977

Brian D. Mason and William I. Hartkopf

Addressing Confusion in Double Star Nomenclature: The Washington

Multiplicity Catalog . . . . . . . . . . . . . . .

William I. Hartkopf and Brian D. Mason

Working Group on Designations and Special Session 3

Helene Dickel and Marion Schmitz

Designation of Multiple-star Components

Andrei Tokovinin

Dynamic versus Static Designation .

Dimitri Pourbaix

Nomenclature for Multiple Systems Containing Close Binaries . . . . . . 1000

C. D. Scarfe

The Viewpoint from Commission $40 \ldots \ldots \ldots$. . . . . . . . 1006

Richard N. Manchester

Nomenclature Scheme in use by the WGESP and a Current List

of Extrasolar Planets . . . . . . . . . . . . . . . . . 1008

Jill C. Tarter

SPS 3: Epilog .

Brian D. Mason and William I. Hartkopf 


\section{SPS 4. EFFECTIVE TEACHING AND LEARNING OF ASTRONOMY}

Chairperson: J. R. Percy

Editor: J. R. Percy and J. M. Pasachoff

A Short Overview of Astronomical Education Carried Out by the International Astronomical Union (IAU) . . . . . . . . . . 1017 Syuzo Isobe

Why Astronomy is Useful and Should be Included in the School

Curriculum . . . . . . . . . . . . . . . . . . 1020 John R. Percy

Astronomy and Mathematics Education . . . . . . . . . . . . . . . 1022

Rosa M. Ros

Astronomy Education Research Down Under . . . . . . . . . . . . . . . 1024

John M. Broadfoot and Ian S. Ginns

A Contemporary Review of K-16 Astronomy Education Research . . . . 1029

Janelle M. Bailey and Timothy F. Slater

Implementing the Astronomy Education Research _ . . . . . . . . . . 1032

Leonarda Fucili

Distance/Internet Astronomy Education . . . . . . . . . . . . . . 1037

David H. McKinnon

Engaging Gifted Science Students through Astronomy . . . . . . . . . . 1041

Robert Hollow

Pre-Service Astronomy Education of Teachers . . . . . . . . . . . . . . . 1044

Mary Kay Hemenway

In-Service Astronomy Education of Teachers . . . . . . . . . . . . . . 1046

Michèle Gerbaldi

Textbooks for K-12 Astronomy . . . . . . . . . . . . . . . . . . . . . . 1048

Jay M. Pasachoff

The Astronomy Education Review: Research, Resources, News, and

Opinions . . . . . . . . . . . . . . . . . 1051

Sidney C. Wolff

Astronomy, Pseudoscience, and Rational Thinking . . . . . . . . . . . 1052

Jayant V. Narlikar

Teaching Astronomy in Other Cultures: Archeoastronomy . . . . . . . . 1055

Julieta Fierro

Astronomy Curriculum for Developing Countries . . . . . . . . . . . 1058 Case Rijsdijk

Fostering Science Education in the Developing Countries . . . . . . . 1061

James C. White II 
What Makes Informal Education (IE) Programs Successful? A Case History: Total Solar Eclipse 2001 — Live from Africa . . . . . . . 1063 Nahide Craig and Isabel Hawkins

The Role of Science Centres and Planetaria . . . . . . . . . . . . 1065 Nick Lomb

Science Education for the New Century-A European Perspective . . . 1068 Claus Madsen

Poster Highlights: Research, Curriculum, and Resources . . . . . . . . . 1069

Author Index . . . . . . . . . . . . . . . . . . . . . 1073 